\title{
Design, Fabrication, and Evaluation of Highly Sensitive Compact Chemical Sensor System Employing a Microcantilever Array and a Preconcentrator
}

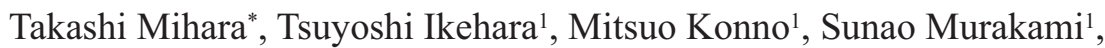 \\ Ryutaro Maeda ${ }^{1}$, Tadashi Fukawa ${ }^{2}$ and Mutsumi Kimura ${ }^{2}$ \\ Future Creation Laboratory, Olympus Corporation, \\ 2-3 Kuboyama-cho, Hachiouji, Tokyo 192-8512, Japan \\ 'National Institute of Advanced Industrial Science and Technology, \\ 1-2 Namiki, Tsukuba, Ibaraki 305-8564, Japan \\ ${ }^{2}$ Faculty of Textile Science \& Technology, Shinshu University, \\ Tokida 3-15-1, Ueda, Nagano 386-8567, Japan
}

(Received January 17, 2011; accepted April 12, 2011)

Key words: chemical sensor, microcantilever, cantilever array, preconcentrator, poly-butadiene, acrylonitrile-co-butadiene, acetone, toluene, xylene

We developed a highly sensitive compact chemical sensor system employing a polymer-coated microcantilever sensor array and a thermal preconcentrator. The design, structure, fabrication, and experiment results are reported here. This sensor system had 1) a sub-ppb detection limit concentrated by a preconcentrator and 2) analytical function by thermal desorption of the preconcentrator and multiple cantilevers (acting as the mass sensor) with different polymers. The preconcentrator contained $0.03 \mathrm{~g}$ of carbon fiber, and absorption/desorption were controlled by the temperature from room temperature to $520^{\circ} \mathrm{C}$. The sample gas was introduced into the preconcentrator using a miniature air pump at a flow rate of $2.0 \mathrm{~L} / \mathrm{min}$. Four silicon microcantilevers in one silicon chip fabricated by micro-electromechanical systems (MEMS) technology were packaged in a ceramic flat package and driven by a PZT actuator plate mounted in the package. Using the 4th vibration mode (resonant frequency: $764 \mathrm{kHz}$ ) of a polybutadiene $(2.52$ $\mu \mathrm{m}$ thick)-coated cantilever, the sensitivity was $514 \mathrm{~Hz} / \mathrm{ppm}$ for toluene and $850 \mathrm{~Hz} /$ ppm for p-xylene with a 5 min preconcentration time. The preconcentration factor and system efficiency of sensing were estimated to be 830 and 0.78 , respectively, for toluene. The estimated detection limit of the sensor system was less than $1 \mathrm{ppb}$ for toluene

"Corresponding author: e-mail: t_mihara@mmc.or.jp 
and p-xylene with a $10 \mathrm{~L}$ sample volume, which was good enough for application to environmental monitoring. Separate detection of the mixed toluene and p-xylene was also achieved in the form of different time peaks during the heating preconcentrator operation.

\section{Introduction}

The development of a chemical sensor system to detect volatile organic compound (VOC) species has been receiving much attention for environmental monitoring and ultrafast medical diagnostics. To date, the widely used sensor to detect VOCs has been the metal oxide semiconductor (MOS) sensor; however, it is difficult to analyze the VOC elements owing to the relatively poor selectivity of compounds. Quartz crystal microbalance (QCM) and surface acoustic wave (SAW) devices with sensing films have also been widely investigated; however, they are also difficult to integrate with electronic devices into a smart sensor. New highly sensitive micromass sensors including a microcantilever have recently been developed. ${ }^{(1-4)}$ However, previous investigations show a somewhat low sensitivity, such as $0.01 \mathrm{~Hz} / \mathrm{ppm}$ for ethanol and $0.1 \mathrm{~Hz} / \mathrm{ppm}$ for toluene.

We have been developing an integrated chemical analysis system with focus on the resonant micromass sensor for this purpose. We have reported studies on the mass sensitivity of the silicon microcantilever with high resonant modes, ${ }^{(5,6)}$ sensing films using copolymer-based elastic polymers, ${ }^{(7)}$ and the first prototype sensor system. ${ }^{(8)}$ We consider that silicon micromass sensors with cantilevers are potential smart sensors because they can be easily integrated with electronic devices to provide intelligent functions in a chemical sensor system. To increase the sensitivity, we used a preconcentrator. To obtain the analytical function, we utilized the desorption characteristics of a heating preconcentrator and cantilever arrays. ${ }^{(9,10)}$ We also reported the estimation of concentration factors and system efficiencies using basic formulas for the cantilever-type chemical sensor system. ${ }^{(11)}$ Several previous investigations to combine a preconcentrator with SAW devices and QCM-type sensors have been reported to date. With the use of the TENAX-TA (polymer beads based on 2,6-diphenyl-p-phenylene oxide by Teijin)-filled preconcentrator and $433 \mathrm{MHz}$ SAW sensor, the detection limit for toluene was reported to be $0.08 \mathrm{ppm}$ and that for ethanol was $33 \mathrm{ppm}$ after 3 min of concentration. ${ }^{(12,13)}$ Another report showed the separation of the components with the thermal preconcentrator combined with QCM devices. ${ }^{(14)}$

In this study, we report the design, fabrication and evaluation results of a newly developed sub-ppb-detectable sensor system possessing both preconcentration and analytical functions. This report contains the detailed design method, system setup, and experimental results for the compact sensor system, as an extension of the previous report. ${ }^{(11)}$ A part of $\S 2$ (including Figs. 1, 3, and 6) was reconstructed from Figs. 1-3 in ref. 11 to maintain the consistency of mathematical notations and to demonstrate the system improvement. 


\section{Design, Materials, and Methods}

\subsection{Design of sensor system}

The potential applications of our chemical sensor system are in environmental monitoring and medicine. The environmental monitoring requires toxic VOC (including toluene and xylene) sensing at extremely low concentration of sub-ppb order for a relatively large air volume (more than $10 \mathrm{~L}$ ). Medical application requires the detection of specific gases, for example, acetone in a few liters of sample to diagnose diabetes. ${ }^{(15)}$ We also require compact size and low-cost operation in addition to on-site measurement in the sensing field. These requirements allow the sensor system to be equipped with an analytical function and a preconcentrator. Thus, our sensor system comprised an air pump, a preconcentrator, and a cantilever array as a sensing device, as shown in Fig. 1. The sample gas was introduced into the preconcentrator by pumping at room temperature. Then, the temperature of the preconcentrator was gradually increased by the heater, and the desorbed gases at a specific temperature were delivered into a sensor chamber with pure nitrogen carrier gas. This desorbed gas was detected by a cantilever array coated with different polymers under controlled temperature. The shifts of frequency appeared at different times, as shown in Fig. 1.

We introduced detailed descriptions of important formulas, including those for the frequency shift of the cantilever sensor, the condition of oscillation for multiple cantilevers, preconcentration and analysis of VOC, the definition of sensitivity, efficiencies and concentration factor of our system, and the detection limit, in addition to those in the previous report. ${ }^{(11)}$ On the basis of these formulas, the sensor system was designed and its performance was evaluated.

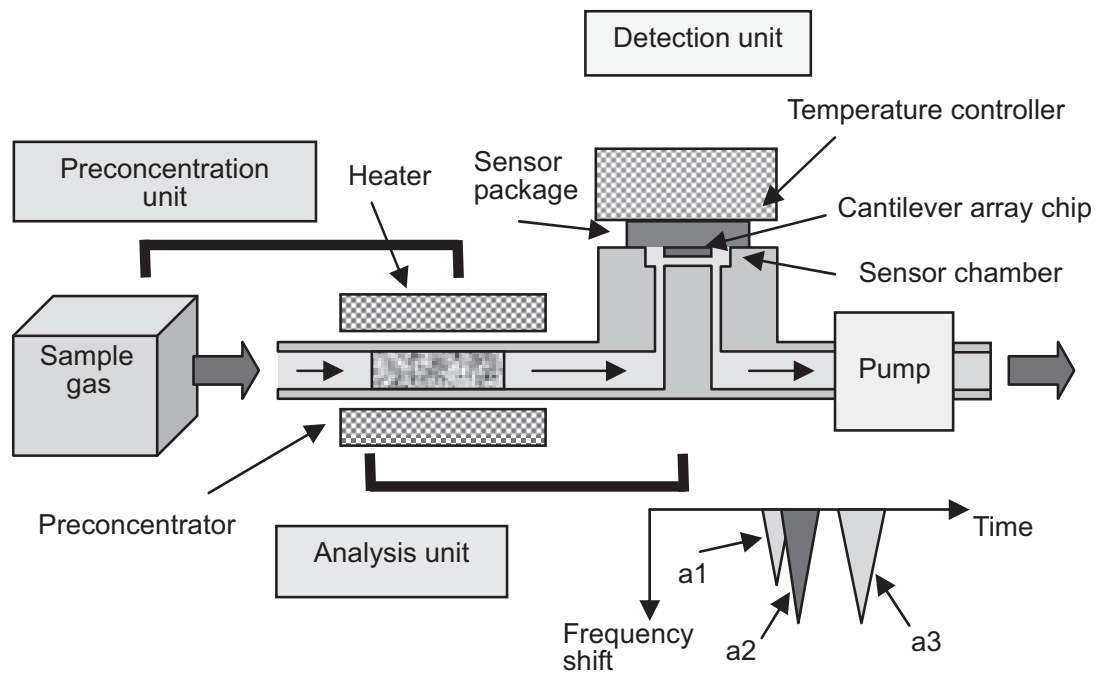

Fig. 1. Sensing concept of our sensor system. 


\subsubsection{Sensitivity of cantilever mass sensor}

We chose a microcantilever as a mass-sensing resonator because its characteristics are well known, its fabrication is well established, and its combination with electronic devices is easy. The resonant frequency of the $n$th flexural mode of resonant frequency $f_{0}$ of a cantilever with length $L_{\mathrm{Si}}$ and thickness $t_{\mathrm{Si}}$ is shown as

$$
f_{0}=k_{n} \frac{t_{\mathrm{Si}}}{L_{\mathrm{Si}}^{2}} \sqrt{\frac{E_{\mathrm{Si}}}{\rho_{\mathrm{Si}}}},
$$

where $E_{\mathrm{Si}}, \rho_{\mathrm{Si}}$, and $k_{n}$ are Young's modulus, the density of silicon, and the coefficient dependent on mode $n$, respectively. ${ }^{(16)}$ After the deposition of polymers that act as VOC sensing materials, the resonant frequency $f_{\mathrm{P}}$ of the $n$th mode vibration is reduced by the change in the cantilever's mass as

$$
f_{\mathrm{P}}=f_{0}-\frac{1}{2} f_{0} \frac{\sigma_{\text {Poly }}}{\sigma_{\mathrm{Si}}+\sigma_{\text {Pad }}},
$$

where $\sigma_{\text {Poly }}, \sigma_{\mathrm{Si}}$, and $\sigma_{\text {Pad }}\left(\mathrm{kg} / \mathrm{m}^{2}\right)$ are the surface densities of the polymer, silicon, and adhesive layer between them, respectively.

The frequency shift $\Delta f_{a}$ of the polymer-coated cantilever after exposure to the diluted VOC " $a$ " is described as

$$
\Delta f_{a}=-\frac{1}{2} f_{\mathrm{P}} \frac{\Delta m_{\mathrm{c}}}{m_{\mathrm{c}}}
$$

where $m_{\mathrm{c}}$ is the mass of the cantilever and $\Delta m_{\mathrm{c}}$ is the change in $m_{\mathrm{c}}$ upon VOC exposure. Equations (2) and (3) are derived from the differential of eq. (1). The $\Delta m_{\mathrm{c}}$ is estimated using a product of $K_{a}$, the concentration of the gas phase, and the volume of polymer as

$$
\Delta m_{\mathrm{c}}=\frac{K_{a} C_{a} M_{a} A_{\mathrm{c}} t_{\text {Poly }}}{0.0224}
$$

where $K_{a}$ is the distribution factor (or $K$-factor) of VOC for the related polymer, which is defined as the ratio of the volume concentrations of VOC in the polymer to that in the surrounding gas in the equilibrium state. $C_{a}$ and $M_{a}$ are the concentration of VOC in mole ratio and the mole weight of diluted VOC, respectively. $A_{\mathrm{c}}$ and $t_{\text {Poly }}$ are the area of the cantilever and the polymer thickness, respectively. The mole volume in the ideal gas state is $0.0224 \mathrm{~m}^{3}$, which was used to transform the mole ratio to the volume ratio. Then $\Delta f_{a}$ is expressed, using the surface densities of the cantilever, as

$$
\Delta f_{a}=-\frac{1}{2} f_{\mathrm{P}} \frac{K_{a} C_{a} M_{a} t_{\text {Poly }}}{0.0224 \cdot 10^{6}\left(\sigma_{\mathrm{Si}}+\sigma_{\mathrm{Pad}}+\sigma_{\mathrm{Poly}}\right)} .
$$


Hence the sensitivity of the cantilever sensor $s_{a}(\mathrm{~Hz} / \mathrm{ppm})$, defined as the ratio of the change in frequency to the concentration in ppm, is shown as

$$
s_{a}=-\frac{1}{2} f_{\mathrm{P}} \frac{K_{a} M_{a} t_{\mathrm{Poly}}}{0.0224 \cdot 10^{6}\left(\sigma_{\mathrm{Si}}+\sigma_{\mathrm{Pad}}+\sigma_{\mathrm{Poly}}\right)} .
$$

The sensitivity is proportional to the resonant frequency, $K_{a}, t_{\text {Poly }}$, and the inverse of the surface density of the cantilever.

\subsubsection{Design of oscillation circuit of cantilever array}

The design of an oscillation circuit of cantilever sensors is described. Figure 2 shows the schematic of the two-cantilever oscillation system. This circuit contains two cantilevers with stress gauges, two front-end amplifiers with gain $g_{i}(i=1,2)$, a multiplexer (MPX), a phase shifter (PS), a bandpass filter (BPF), and a PZT plate as an actuator. First, we explain the design for one cantilever sensor. The driving signal $p_{i}(t)$ fed to the PZT actuation plate is expressed using the amplitude $P_{0 i}$, the driving frequency $f_{\mathrm{PZT}}$, and the phase $\chi_{i}$ of driving signal as

$$
p_{i}(t)=P_{0 i} \sin \left(2 \pi f_{\mathrm{PZT}} t+\chi_{i}\right) .
$$

When MPX selects the $i$ th cantilever, the signal $u_{i}(t)$ of the piezoresistive gauge with the specific vibration mode of the $i$ th cantilever is expressed with the amplitude $U_{0 i}$, the resonant frequency $f_{i}$, and the phase shift $\varphi_{i}$ as

$$
u_{i}(t)=U_{0 i} \sin \left\{2 \pi\left[f_{i}\left(1+\beta_{i} \Delta T_{\mathrm{c}}\right)\right] t+\varphi_{i}+\delta \varphi_{i}\right\} .
$$

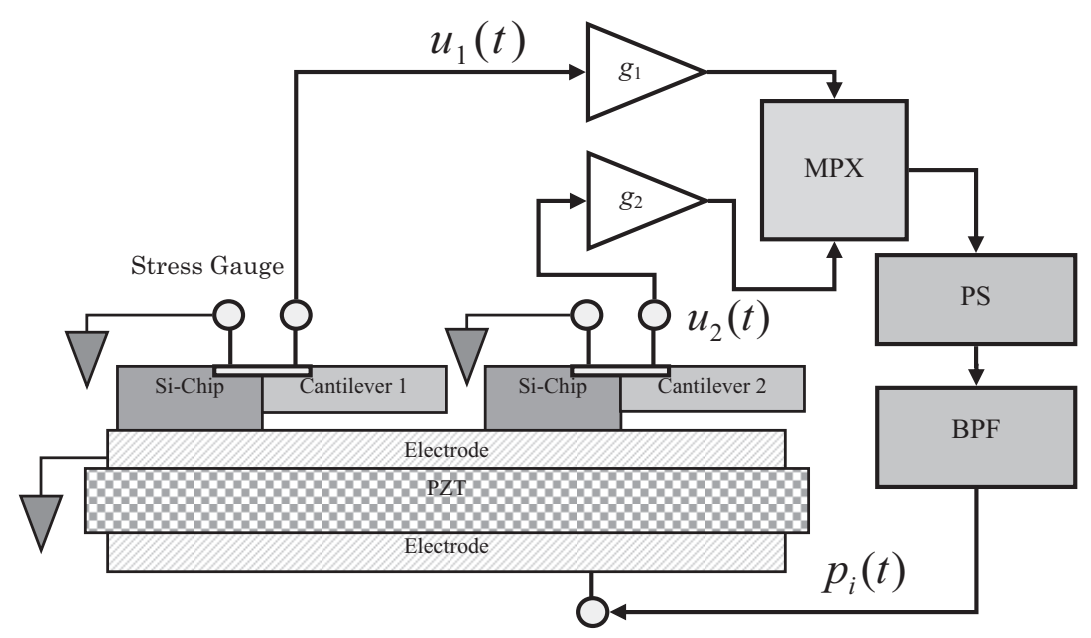

Fig. 2. Schematic of two-cantilever oscillation system. 
Equation (8) also includes the temperature coefficient $\beta_{i}$, the change in cantilever temperature $\Delta T_{\mathrm{c}}$, and the phase variation $\delta \varphi_{i}$. The temperature dependence of frequency produced by $\beta_{i}$ is a major cause of the frequency drift and should be compensated by the measurement of chip temperature. ${ }^{(17)}$ The $\delta \varphi_{i}$ produces the short-range variation of frequency that determines the detection limit of chemical sensors.

We designed the oscillation circuit of the cantilever subject to the following three items.

(1) The $f_{\mathrm{PZT}}$ is adjusted by the central frequency of BPF to match the resonant frequency of the cantilever as

$$
f_{\mathrm{PZT}}=f_{i}\left(1+\beta_{i} \Delta T_{\mathrm{c}}\right)
$$

(2) The total gain $G_{i}$ of the circuit, including $g_{i}$ of the front-end amplifier, is set to be larger than the ratio of $P_{0 i}$ to $U_{0 i}$ :

$$
\frac{P_{0 i}}{U_{0 i}} \leq G_{i}
$$

The $g_{i}$ is adjusted to the specific value for the $i$ th cantilever and $G_{i}$ is automatically adjusted by AGC to an adequate value of $G_{i}=P_{0 i} / U_{0 i}$ when stable oscillation occurs.

(3) The phase $\pi_{i}$ of PS is adjusted such that the sum of phases $\varphi_{i}, \chi_{i}, \pi_{i}$, and a phase $\theta_{i}$ of other circuits including the amplifier, BPF, and MPX is a multiple of $2 \pi$ as

$$
\varphi_{i}+\chi_{i}+\pi_{i}+\theta_{i}=2 \pi I
$$

where $I$ is an integer. In actual experiment, the central frequency of BPF is adjusted to obtain the maximum values of $P_{0 i}$. After that, $\pi_{i}$ of PS is adjusted to obtain the maximum values of $U_{0 i}$. Then the stable resonant vibration of the cantilever occurs. Note that the $G_{i}$ varies with many mechanical parameters including the efficiency/coupling factors of PZT, the piezoresistive gauge, and the adhesive glue.

We now explain the case of the cantilever array. We applied electrical multiplexing to drive multiple cantilevers, because the oscillation circuit became complicated, making it difficult to obtain stable oscillation. After the switching of the sensor signal from $i$ to $i+1$ using MPX, $u_{i}(t)$ is altered to $u_{i+1}(t)$ by the specific $U_{0 i+1}, f_{i+1}$, and $\varphi_{i+1}$ for each cantilever, as shown in eq. (8). The design and oscillation conditions should also be satisfied on the (i+1)th cantilever, as shown in eqs. (9) to (11). We found that $U_{0 i}, f_{i}$, and $\varphi_{i}$ for each cantilever with polymer are not the same, and these values should be adjusted after multiplexing. Then, we utilized a presetting circuit with memory, as described in $\S$ 2.2.3.

\subsubsection{Preconcentration}

We applied a preconcentrator to improve the detection limit of the system at the ppb level. Figure 3 shows the schematic diagram of the flow of the gas sample from the sample bag to the sensor. The VOC gas with component " $a$ " and mass $m_{a}(\mathrm{~kg})$ in the gas 


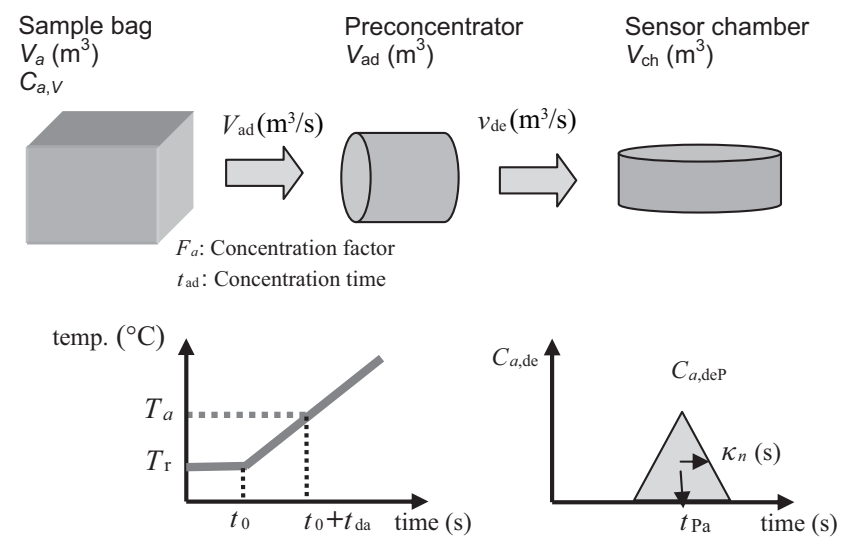

Fig. 3. Block diagram of gas flow from sample bag to sensor.

state is diluted in volume $V_{a}\left(\mathrm{~m}^{3}\right)$ with concentration $C_{a, V}$, as shown in Fig. 3. When the gas of $V_{a}$ is introduced to the preconcentrator of $V_{\text {ad }}\left(\mathrm{m}^{3}\right)$ with flow of a volume velocity of $v_{\text {ad }}\left(\mathrm{m}^{3} / \mathrm{s}\right)$, component " $a$ " will be adsorbed in the adsorption material. The mass of component " $a$ " in the preconcentrator $m_{a, \text { ad }}(\mathrm{kg})$ is reduced by the preconcentrator efficiency $\eta_{a}$ as

$$
m_{a, \text { ad }}=\eta_{a} m_{a}=\eta_{a} \frac{M_{a} V_{a} C_{a, V}}{0.0224}
$$

Then, $\left(1-\eta_{a}\right)$ represents for the part that escapes from the preconcentrator when sample gas is adsorbed.

\subsubsection{Sensing response of VOC by sensor system with preconcentrator}

The adsorbed VOC in the preconcentrator is desorbed by heating and streamed to the sensor chamber by high-purity nitrogen carrier gas with a volume velocity of $v_{\text {de }}\left(\mathrm{m}^{3} / \mathrm{s}\right)$, as shown in Fig. 3. The peak time $t_{\mathrm{Pa}}(s)$ of component " $a$ " measured with the sensor is presented as

$$
t_{\mathrm{Pa}}=t_{0}+\frac{T_{a}-T_{\mathrm{r}}}{r_{\mathrm{t}}}+\frac{S_{\mathrm{t}} L_{\mathrm{t}}}{v_{\mathrm{de}}}+\frac{V_{\mathrm{ch}}}{v_{\mathrm{de}}},
$$

where $t_{0}(s)$ is the start time of heating, as shown in Fig. 3. The second term stands for the time taken to reach desorption $t_{\mathrm{da}}(s)$, in which $T_{\mathrm{r}}, T_{a}\left({ }^{\circ} \mathrm{C}\right)$, and $r_{\mathrm{t}}\left({ }^{\circ} \mathrm{C} / \mathrm{s}\right)$ are room temperature, the desorption temperature of VOC " $a$," and the heating rate of the preconcentrator, respectively, as shown in Fig. 3. The third term is the delivery time from the preconcentrator to the sensor chamber by the carrier gas, in which $S_{\mathrm{t}}\left(\mathrm{m}^{2}\right)$ is 
the cross-sectional area of the gas tube, $L_{\mathrm{t}}(\mathrm{m})$ the length of the tube, and $v_{\mathrm{de}}\left(\mathrm{m}^{3} / \mathrm{s}\right)$ the volume velocity of the carrier gas. The fourth term is the filling time of VOC in the sensor chamber with volume $V_{\mathrm{ch}}\left(\mathrm{m}^{3}\right)$. If the sample gas is a mixture of several VOCs, multiple peaks are observed because of the difference in $T_{a}$ among VOCs. The $T_{a}$ is determined as the extrapolation value at $y$-axis of $1 / v_{\mathrm{de}}$ dependence of $t_{\mathrm{Pa}}$.

The desorption response of absorbed gas is explained on the basis of the theory of temperature-programmed desorption (TPD). ${ }^{(18)}$ The time $t$ dependence of desorption molecules $N_{\mathrm{d}}(t)$ (molecules/s) is expressed with the absorbed surface amount $\Omega_{z}$ (molecules $/ \mathrm{cm}^{2}$ ), the reaction order of desorption $z$, the absorption rate $\gamma_{z}$ (molecules/s), the desorption energy $E_{a}(\mathrm{eV})$, and the Boltzmann constant $k_{\mathrm{B}}$ as

$$
N_{\mathrm{d}}(t)=-\frac{\mathrm{d} \Omega_{z}}{\mathrm{~d} t}=\gamma_{z} \Omega_{z}^{z} \exp \left[\frac{E_{a}}{k_{\mathrm{B}}\left(T_{a}+273.15\right)}\right] .
$$

However, $\Omega_{z}$ in eq. (14) is solved by the numerical integration with adsorption temperature, and $N_{\mathrm{d}}(t)$ is solved by the numerical differential, which is too complicated to fit an actual sensing response curve to analyze the multiple components. Therefore, we use the linear combination of several Gaussian functions as

$$
\Delta f_{\text {mix }}(t)=\Delta f_{0 \text { mix }}+\sum_{j=1}^{N} \Delta f_{\mathrm{Pa}, j} \exp \left[-\frac{\left(t-t_{\mathrm{Pa}, j}\right)^{2}}{\kappa_{a, j}^{2}}\right],
$$

to explain the actual sensor response. Hence, $\Delta f_{\text {mix }}(t), \Delta f_{0 \text { mix }}, \Delta f_{\mathrm{Pa}, j}, t_{\mathrm{Pa}, j}$, and $\kappa_{a, j}$ are the response of frequency shift by mixed VOCs, the baseline of $\Delta f_{\text {mix }}(t)$, the peak value of frequency shift for the $j$ th component, the peak time for the $j$ th (or " $a$ ") component, and the standard deviation for the $j$ th (or " $a$ ") component, respectively. Note that $t_{\mathrm{Pa}}$ in eq. (13) is equal to $t_{\mathrm{Pa}, j}$ when $j=1$.

The final sensing mass $m_{a, \mathrm{de}}(\mathrm{kg})$ of component " $a$ " detected by the sensor is calculated with the desorption response as

$$
m_{a, \mathrm{de}}=\frac{M_{a}}{0.0224} v_{\mathrm{de}} \sum_{\Delta t_{\mathrm{de}}} \Delta t_{\mathrm{de}} C_{a, \mathrm{de}}(t)=\frac{M_{a}}{0.0224} v_{\mathrm{de}} \sum_{\Delta t_{\mathrm{de}}} \Delta t_{\mathrm{de}} s_{a} \Delta f_{a}(t)
$$

where $\Delta t_{\mathrm{de}}(s)$ is time interval of sensing, and $C_{a, \mathrm{de}}(t)$ is the observed concentration of VOC by the cantilever sensor at time $t$. This $m_{a, \mathrm{de}}$ is a part of the mass $m_{a, \text { ad }}$ of absorbed VOC in the preconcentrator; then, $m_{a, \mathrm{de}}$ is presented by another efficiency $\xi_{a}$ as

$$
m_{a, \mathrm{ad}}=\xi_{a} m_{a, \mathrm{ad}}=\xi_{a} \eta_{a} m_{a}
$$

We can evaluate the total efficiency $\xi_{a} \eta_{a}$ of the system by taking the ratio of $m_{a, \mathrm{de}}$ to $m_{a}$. The concentration factor $F_{a, V}$ of a sensor system is defined using the ratio of $C_{a, \mathrm{deP}}$ (peak value of $C_{a, \mathrm{de}}(t)$ in Fig. 3) to $C_{a, V}$ as 


$$
F_{a, V}=\frac{C_{a, \mathrm{deP}}}{C_{a, V}}
$$

which is proportional to $V_{a}$. In practical use, the sample volume is determined from the volume of the sample bag or the time of aspiration $t_{\mathrm{ad}}(s)$ into the preconcentrator by an air pump. Then, we defined the system sensitivity $S_{a, 10 \mathrm{~L}}\left(\mathrm{~Hz} / \mathrm{ppm}\right.$ when $\left.V_{a}=10 \mathrm{~L}\right)$ as

$$
S_{a, 10 \mathrm{~L}}=\frac{\Delta f_{a, 10 \mathrm{~L}}}{C_{a, 10 \mathrm{~L}}}=F_{a, 10 \mathrm{~L}} S_{a},
$$

where $\Delta f_{a, 10 \mathrm{~L}}$ and $C_{a, 10 \mathrm{~L}}$ are the measured frequency shift and the concentration when $V_{a}=10 \mathrm{~L}$, and $F_{a, 10 \mathrm{~L}}$ is the measured concentration factor when $V_{a}=10 \mathrm{~L}$.

Finally, the detection limit $D L_{a, 10 \mathrm{~L}}\left(\mathrm{ppb}\right.$ when $V_{a}=10 \mathrm{~L}$ ) is derived from the standard variation $\lambda$ of $\Delta f_{a}$ in a short time and $S_{a, 10 \mathrm{~L}}$ as

$$
D L_{a, 10 \mathrm{~L}}=\frac{3 \lambda}{10^{-3} S_{a, 10 \mathrm{~L}}}=\frac{3 \lambda}{10^{-3} F_{a, 10 \mathrm{~L}} s_{a}},
$$

where $10^{-3}$ is used to transform ppm to $\mathrm{ppb}$. To obtain a low detection limit, efforts to reduce $\lambda$, including the high quality factor of the resonator, and to reduce electric noise are essential, in addition to the high sensitivity.

We expressed the important formulas of our sensor system described above for the purpose of designing the sensor system. We used eqs. (1) to (6) to analyze and design a cantilever sensor, eqs. (7) to (11) to design oscillation circuits and temperature compensation, and eqs. (12) to (20) to design the operation parameters and analyze the performance.

\subsection{System components}

\subsubsection{Cantilever sensors and packaging}

Silicon cantilevers were fabricated by microfabrication techniques from an SOI wafer. ${ }^{(5)}$ The length, width, and thickness of the cantilever were 500,100 , and $5 \mu \mathrm{m}$, respectively. A set of bridged piezoresistive stress gauges was formed at the root of the cantilever from the p-type layer in the n-type active layer employing boron implantation and thermal diffusion. The substrate under the cantilever was removed by deep RIE from the backside to eliminate the squeezed air damping effect. A gold pattern was formed on the upper surface of the cantilever to obtain an adequate adhesion characteristic with polymers. Eight cantilevers in one silicon chip were packaged in a ceramic flat package and driven by a PZT actuator plate mounted in the package. The silicon chip was adhered to the PZT plate with epoxy, and the PZT plate was mounted on the flat package with epoxy at the four corners and Ag paste at the center, as shown in Fig. 4(a). Four of the eight cantilevers were wire bonded and used as sensors. Figure 4(a) also shows the temperature controller attached to the package. 


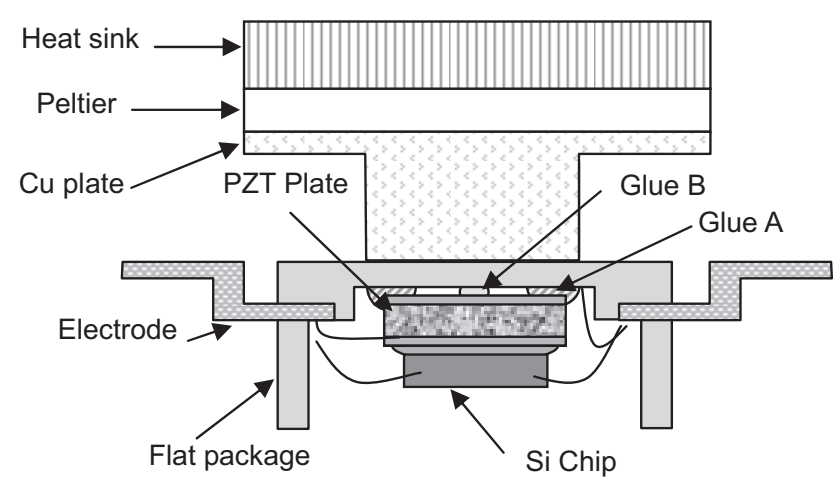

(a)

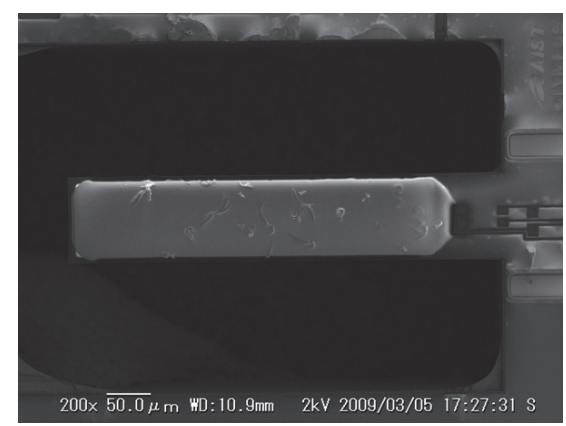

(b)

Fig. 4. (a) Flat package of cantilever chip with PZT plate and a temperature controller, (b) SEM image of PBD-coated cantilever.

\subsubsection{Polymer (sensing material)}

Two types of polymer among the elastomer-type materials were used as VOC sensing films in this study. One was poly-butadiene (PBD), which is sensitive to aromatic VOC gases including toluene and xylene. The other was poly(acrylonitrile-co-butadiene) (PAB), which is sensitive to alcohol and acetone. We found that the PBD and PAB films showed fast response to adsorption and desorption while these films were exposed to various VOCs. ${ }^{(7,19)}$ These thin films were deposited on the cantilever by the microdispensing technique. The thicknesses of the PBD and PAB films were controlled from 300 to 2,500 $\mathrm{nm}$ by varying the duration of dispensing. A SEM image of the PBD-coated cantilever demonstrates that flat and smooth surfaces were obtained, as shown in Fig. 4(b).

\subsubsection{Oscillation circuit}

The signal from the bridged piezoresistive stress gauge on each cantilever was amplified by a front-end differential amplifier (A), then selected by an electrically programmed MPX and oscillated using a feedback circuit, as shown in Fig. 5. The oscillation circuit consisted of an automatic gain controller (AGC), a PS and a secondorder BPF. The $f_{i}$, amplitude $U_{0 i}$, and phase $\varphi_{i}$, as presented in eq. (8) for each cantilever $(i=1$ to 4$)$, were greatly varied among cantilevers owing to the difference in mechanical characteristics depending on the coated materials, the site of the cantilever in a chip, and the method of PZT mounting, as shown in Fig. 4(a). Therefore, two parameters, namely, 1) the amount of feedback phase shift $\pi_{i}$ in eq. (11) and 2) $f_{i}$ for the $i$-th cantilever, were stored in the memory on the oscillation circuit board and used when multiplexing to achieve oscillation under the best conditions. Hence, the amplitude $U_{0 i}$ was automatically adjusted with AGC. Figure 5 shows the additional AD and DA converters and random access memories (RAMs) in conjunction with PS and BPF to preset and store the feedback phase and the resonance frequency. Thanks to these preset circuits, we could oscillate cantilevers of various sizes, modes and polymer materials. 


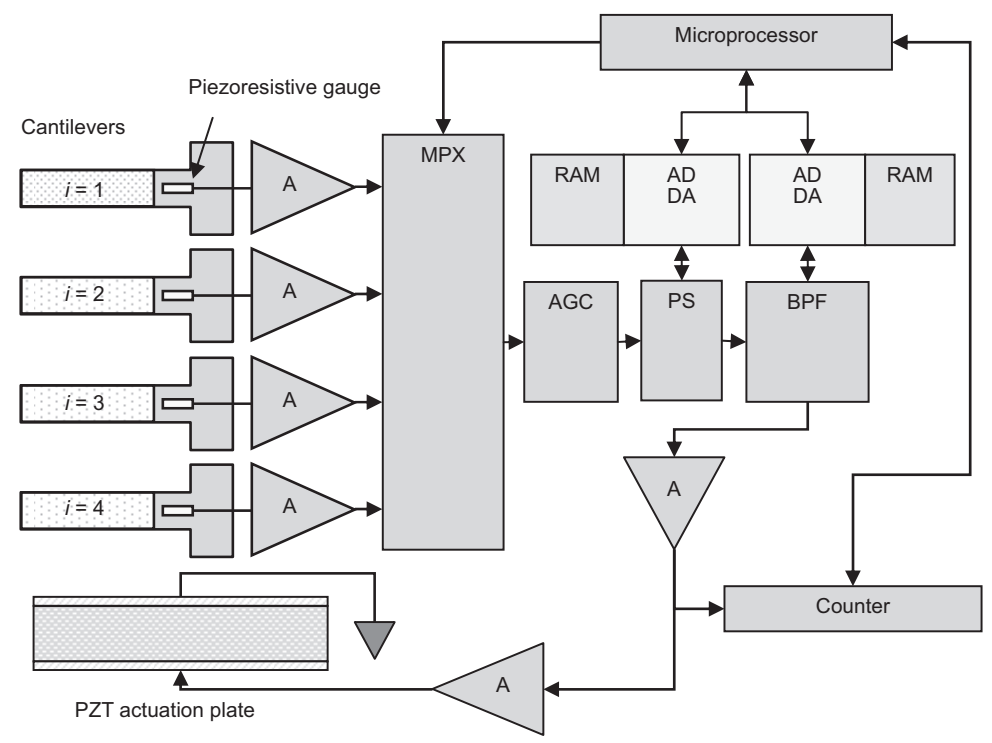

Fig. 5. Block diagram of oscillation circuit with phase and resonant frequency preset at MPX switching.

\subsubsection{Preconcentrator}

A preconcentrator was made of a 6.35 -mm-diameter stainless-steel tube, in which $0.03 \mathrm{~g}$ of carbon fiber (Kuraray Chemical: maximum surface area of about 2,500 $\mathrm{m}^{2} / \mathrm{g}$ ) was filled, as shown in Fig. 6. A 1-mm-diameter sheathed heater was wound on the tube with thermal insulation material. This preconcentrator, which is capable of being heated up to $600^{\circ} \mathrm{C}$, was installed at the inlet of the sensor chamber to concentrate a sample gas in the sample bag or environmental air directly. To separate the mixed VOCs, the temperature of the preconcentrator was gradually increased at $r_{\mathrm{t}}=1.0$ to $1.3^{\circ} \mathrm{C} / \mathrm{s}$ using the programmed DC power supply.

\subsubsection{Total sensor system structure}

Figure 7(a) shows the sensor system including an air pump, a preconcentrator, and a sensor chamber. A Peltier device with an electric fan was installed in the sensor chamber to keep the temperature of the cantilever sensor constant. The temperature of the cantilever sensor was measured using a $\mathrm{p}-\mathrm{n}$ junction diode fabricated in the same chip. ${ }^{(17)}$ The size of the total system was $280 \times 200 \times 80 \mathrm{~mm}^{3}$. Figure 7 (b) also shows an electronics case including three electrical circuit boards for oscillation, control, and power units. These circuits included two R8c microcomputers (Renesas Electronics Corporation), and all control commands and measured data were shared with a personal computer (PC) via a universal serial bus (USB) interface. Measurement and analysis 


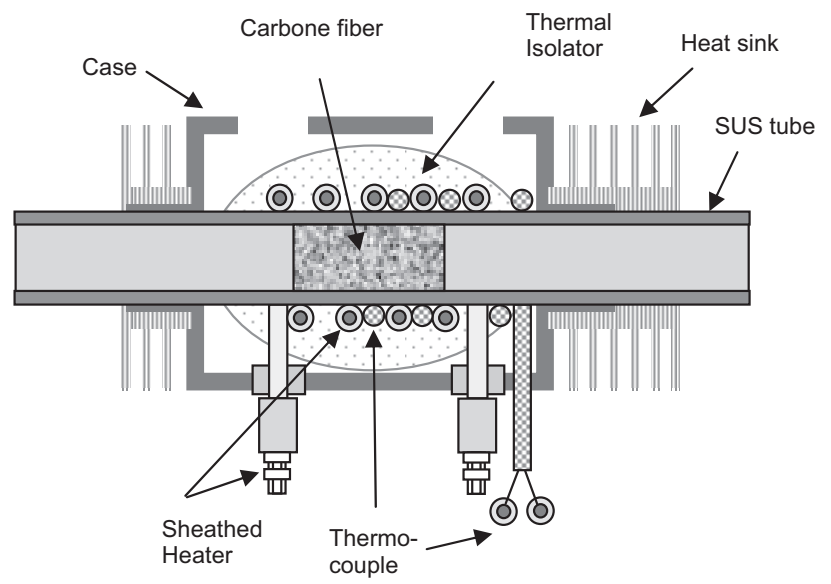

(a)

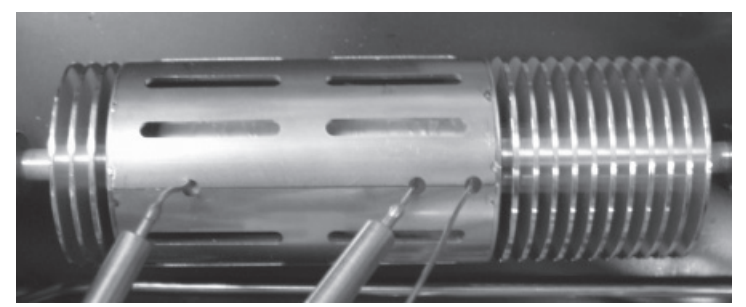

(b)

Fig. 6. Preconcentrator: (a) cross-sectional schematic and (b) photograph of covered preconcentrator.

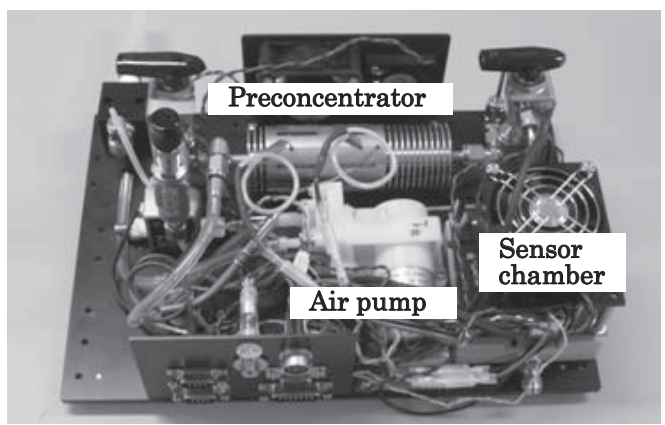

(a)

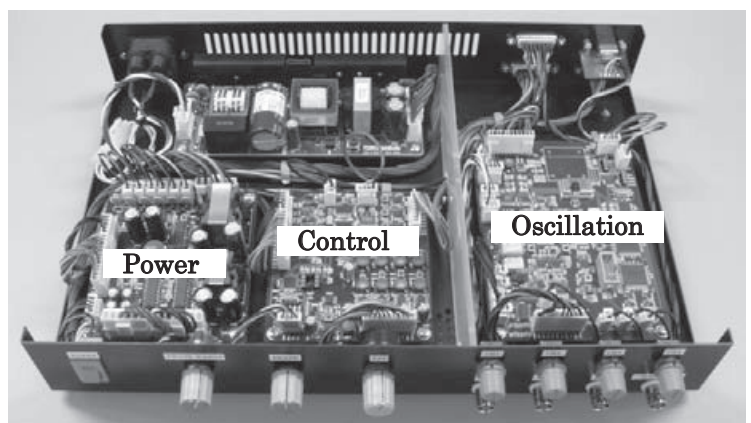

(b)

Fig. 7. Photographs of our sensor system: (a) sensor system including air pump, preconcentrator, and sensor chamber; (b) electrical units with three circuit boards.

software was programmed with Visual Basic, in which several design formulas in this paper were implemented to evaluate system parameters. Figure 8(a) shows the airflow diagram of our sensor system shown in Fig. 7(a), in which three-port manual valves (V), a mass flow meter (MFM) and a needle valve are shown. Figure 8(b) shows two measurement steps of an actual application: (1) in preconcentration, a pump pulls the sample gas though a preconcentrator at room temperature, and (2) in detection and analysis, pure nitrogen carrier gas pushes the desorbed VOCs from the heating preconcentrator into the sensor chamber, where a needle valve maintains volume velocity $v_{\mathrm{de}}$ of the carrier gas constant with MFM monitoring. 
1) Preconcentration step

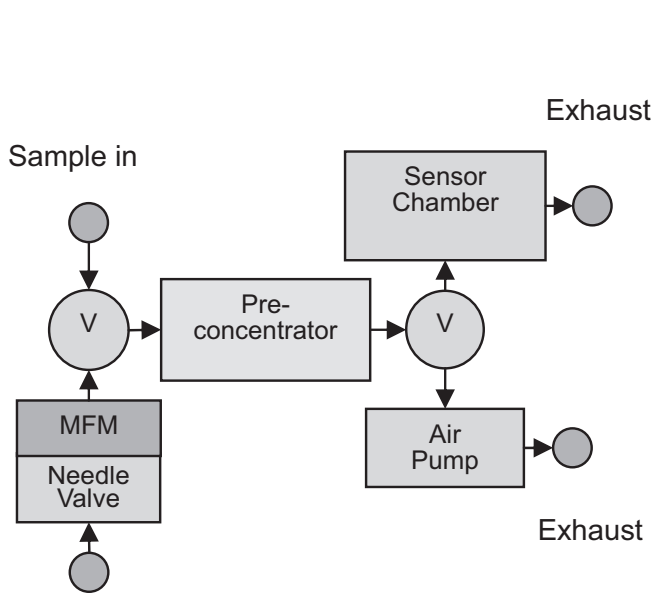

Carrier gas

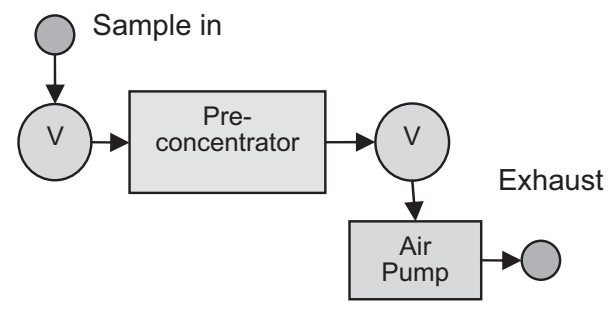

2) Detection step

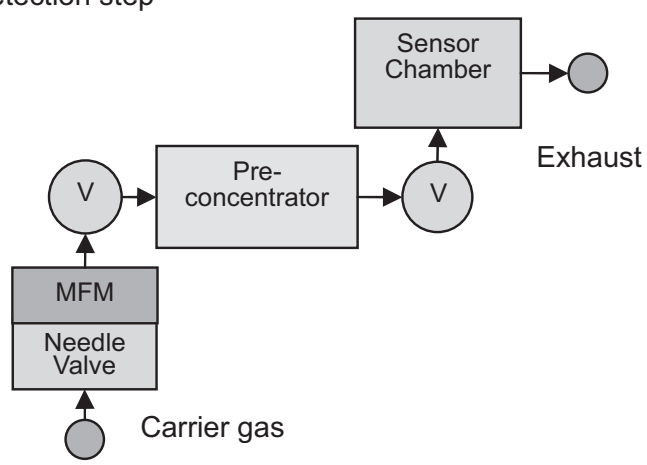

(b)

Fig. 8. (a) Configuration of our sensor system. (b) VOC flow in actual operation: 1) preconcentration of sample gas, and 2) analysis and detection.

\section{Results}

\subsection{Performance of polymer-coated cantilever without preconcentrator}

\subsubsection{Estimations of thickness of polymers}

First, we estimated the thicknesses of polymers as the sensing material. The prepared samples have been described in $\S 2.2 .1$ and $\S 2.2 .2$, and the polymers were PAB and PBD. We evaluated the 4 th mode of resonant frequency $f_{0}$ (before coating) and $f_{\mathrm{P}}$ (after coating) to determine the thickness using eqs. (1) to (2). The thickness $t_{\mathrm{Si}}$ was determined using $f_{0}$ in eq. (1), and $t_{\text {Poly }}$ was calculated using eq. (2) from the difference between $f_{\mathrm{P}}$ and $f_{0}$ shown in Table 1 . Thus, the thicknesses of PBD and PAB were evaluated to be 2,520 and $640 \mathrm{~nm}$, respectively.

\subsubsection{Estimations of sensitivity and K-factor}

Then, we evaluated the $C_{a}$ dependence of $\Delta f_{a}$ for several VOCs to determine the $K_{a}$. Figure 9 shows $\Delta f_{a}$ for four VOCs without a preconcentrator: (a) 640 -nm-thick PAB thin film and (b) 2,520-nm-thick PBD thin film. The drift componet of $\Delta f_{a}$ was compensated 
Table 1

Sensitivities and K-factors for VOCs for PAB- and PBD-coated cantilevers.

\begin{tabular}{|c|c|c|c|c|c|c|c|c|c|c|c|c|}
\hline \multirow{2}{*}{ Polymer } & \multirow{2}{*}{$\begin{array}{c}f_{0} \\
(\mathrm{kHz})\end{array}$} & \multirow{2}{*}{$\begin{array}{c}f_{\text {poly }} \\
(\mathrm{kHz})\end{array}$} & \multirow{2}{*}{$\begin{array}{c}t_{\mathrm{Si}} \\
(\mu \mathrm{m})\end{array}$} & \multirow{2}{*}{$\begin{array}{c}t_{\text {Poly }} \\
(\mathrm{nm})\end{array}$} & \multicolumn{2}{|c|}{ Acetone } & \multicolumn{2}{|c|}{ 1-Propanol } & \multicolumn{2}{|c|}{ Toluene } & \multicolumn{2}{|c|}{ p-Xylene } \\
\hline & & & & & $S_{\text {ace }}$ & $K_{\text {ace }}$ & $S_{\mathrm{pnl}}$ & $K_{\mathrm{pnl}}$ & $s_{\mathrm{tol}}$ & $K_{\text {tol }}$ & $S_{\text {pxy }}$ & $K_{\mathrm{pxy}}$ \\
\hline PAB & 911.4 & 885.2 & 4.87 & 640 & 0.036 & 605 & 0.15 & 2,380 & 0.25 & 2,650 & 0.63 & 5,800 \\
\hline PBD & 862.5 & 764.3 & 4.69 & 2,520 & 0.025 & 141 & 0.076 & 420 & 0.62 & 2,200 & 1.85 & 5,800 \\
\hline
\end{tabular}

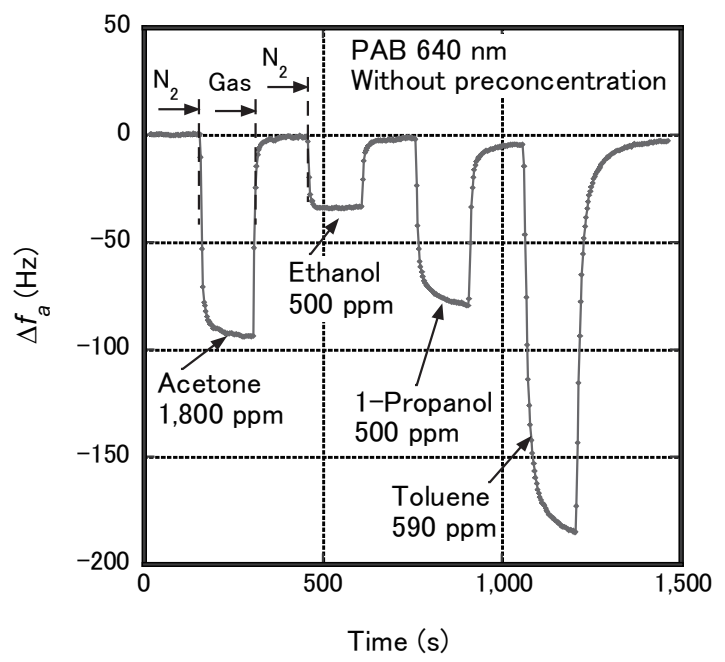

(a)

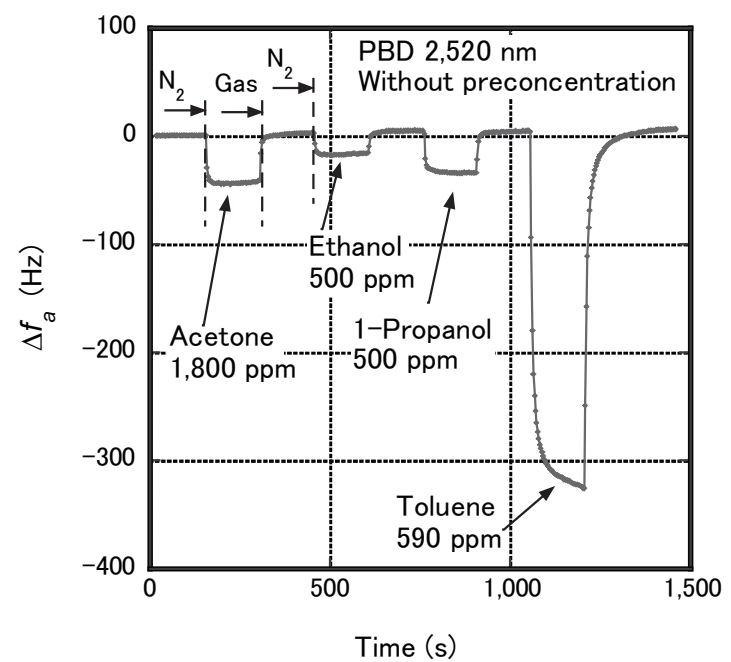

(b)

Fig. 9. Frequency shift of polymer-coated cantilever for four VOCs without preconcentration: (a) 640-nm-thick PAB thin film, (b) 2,520-nm-thick PBD thin film.

by $\beta_{i}\left(183 \mathrm{ppm} /{ }^{\circ} \mathrm{C}\right.$ for $\mathrm{PAB}, 78 \mathrm{ppm} /{ }^{\circ} \mathrm{C}$ for $\left.\mathrm{PBD}\right)$ and $\Delta T_{\mathrm{c}}$ measured using an on-chip p-n diode, ${ }^{(17)}$ as in eq. (8). The diluted $1,800 \mathrm{ppm}$ acetone, $500 \mathrm{ppm}$ ethanol, $500 \mathrm{ppm}$ 1-propanol, and $590 \mathrm{ppm}$ toluene gases were injected in turn into the sensor chamber separated by pure nitrogen gas. These two polymers had different characteristics of sensitivity among the four VOCs: PBD has high selectivity for toluene and PAB had a relativity high sensitivity for acetone and alcohol. Figure 10 shows the concentration dependence of $\Delta f_{a}$ among several VOCs. Then, we estimated the sensitivity $s_{a}$ among VOCs, as shown in Table 1. Using eq. (6) with $s_{a}$, we also calculated the $K_{a}$ for several VOCs, as shown in Table 1. For example, $K_{\text {ace }}$ (for acetone) was 605 for PAB and 141 for PBD. $K_{\text {tol }}$ (for toluene) was 2,650 for PAB and 2,200 for PBD. Note that $K_{\text {pxy }}$ (for p-xylene) values for PAB and PBD was an extremely large value of 5,800. Since these $K_{a}$ values are independent of $t_{\mathrm{Poly}}, f_{\mathrm{P}}$, and $C_{a}$, and $K_{a}$ is an adequate parameter of gas adsorption selectivity for polymers. 


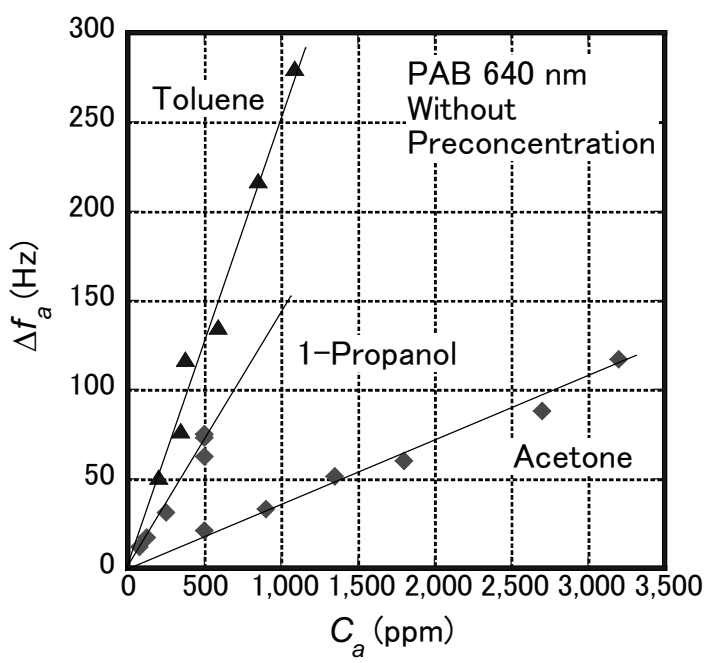

(a)

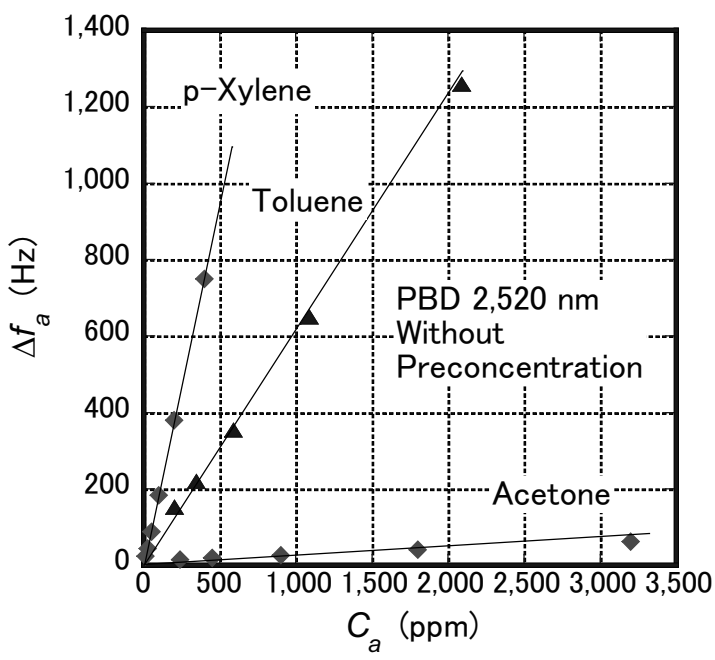

(b)

Fig. 10. Frequency shift of polymer-coated cantilever for four VOCs without preconcentration: (a) 640-nm-thick PAB thin film, (b) 2,520-nm-thick PBD thin film.

\subsection{Performance of sensor system}

\subsubsection{Sensor signal from preconcentrated mixed VOCs}

To explain the analytical function of our sensor system, we evaluated the response of $\Delta f_{a}$ from preconcentrated mixed VOCs. Two types of cantilever sensors, namely, 1) PAB of different thicknesses for acetone-toluene mixed gas and 2) PBD of different thicknesses for toluene-xylene mixed gas, are prepared and attached to a sensor system, as shown in Figs. 7(a) and 7(b). The typical conditions were as follows: $V_{a}=10 \mathrm{~L}\left(10^{-2} \mathrm{~m}^{3}\right)$, $v_{\mathrm{ad}}=2.0 \mathrm{~L} / \mathrm{min}\left(3.3 \times 10^{-5} \mathrm{~m}^{3} / \mathrm{s}\right), v_{\mathrm{de}}=17.2 \mathrm{sccm}\left(2.86 \times 10^{-7} \mathrm{~m}^{3} / \mathrm{s}\right), T_{\mathrm{r}}=27^{\circ} \mathrm{C}$, and maximum preconcentrator temperature of $520^{\circ} \mathrm{C}$. Figure 11 (a) shows the frequency shift $\Delta f_{a, 10 \mathrm{~L}}$ caused by a desorbed mixed gas of acetone $\left(C_{\text {ace, } 10 \mathrm{~L}}=1.8 \mathrm{ppm}\right)$ and toluene $\left(C_{\text {tol, } 10 \mathrm{~L}}=0.69 \mathrm{ppm}\right)$, measured using two PAB-coated cantilever sensors with different thicknesses $\left(t_{\text {Poly }}=330\right.$ and $\left.640 \mathrm{~nm}\right)$. The response curves had two distinct peaks corresponding to acetone and toluene. Figure 11(b) shows $\Delta f_{a, 10 \mathrm{~L}}$ due to the desorbed mixed gas of toluene $\left(C_{\mathrm{tol}, 10 \mathrm{~L}}=0.69 \mathrm{ppm}\right)$ and p-xylene $\left(C_{\mathrm{pxy}, 10 \mathrm{~L}}=0.5 \mathrm{ppm}\right)$ measured using three PBD-coated cantilever sensors with different thicknesses $\left(t_{\text {Poly }}=620,1,060\right.$, and $2,520 \mathrm{~nm}$ ). Three response curves with two peaks were also distinguished, and the peak values of $\Delta f_{a, 10 \mathrm{~L}}$ for the different thicknesses were proportional to $t_{\text {Poly }}$. We could evaluate the response of the desorption gas at the same time using a multiple cantilever system in a preset method for phase and frequency, as shown in Fig. 5. 


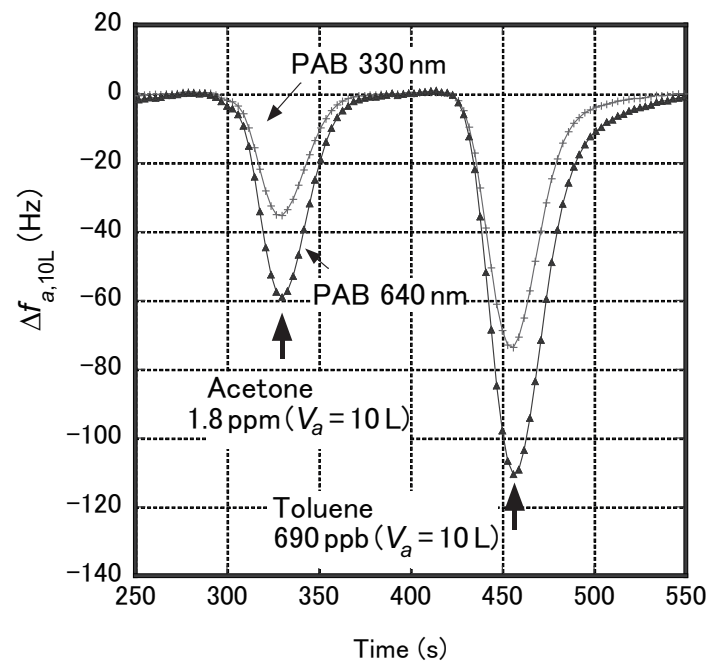

(a)

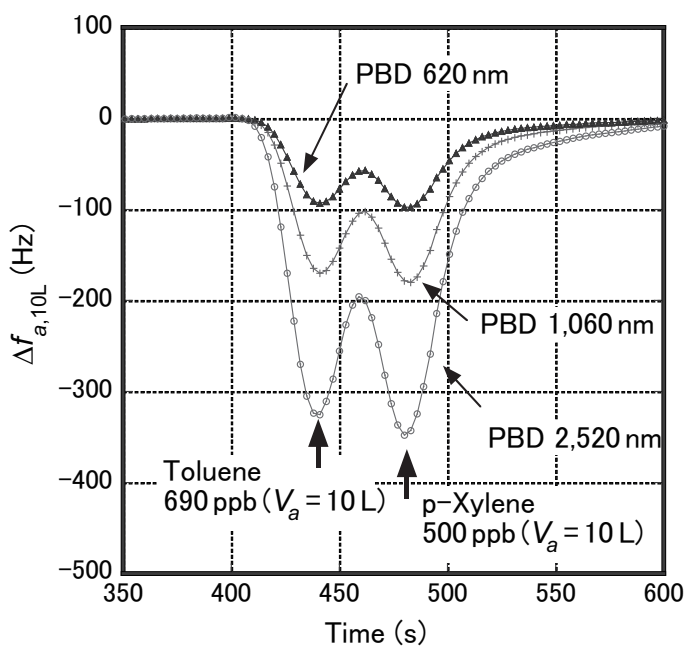

(b)

Fig. 11. Desorption responses of polymer-coated cantilevers for VOCs with preconcentrator: (a) acetone and toluene using PAB-coated sensor, (b) toluene and p-xylene using PBD-coated sensor.

\subsubsection{Estimations of preconcentration factor, efficiency, and desorption temperature}

To estimate several important parameters, including preconcentration factor $F_{a, 10 \mathrm{~L}}$ and system efficiency $\xi_{a} \eta_{a}$ shown in eqs. (16) to (18), the $v_{\text {de }}$ dependences of $\Delta f_{a}$ was evaluated. The prepared samples were described in $\S 2.2 .1$ and $\S 2.2 .2$, and the polymers were PAB $\left(t_{\text {Poly }}=640 \mathrm{~nm}\right)$ and $\operatorname{PBD}\left(t_{\text {Poly }}=2,520 \mathrm{~nm}\right)$. Figure 12 shows the $v_{\mathrm{de}}$ dependences of $F_{a, 10 \mathrm{~L}}$ and $\xi_{a} \eta_{a}$ of (a) acetone for PAB and (b) toluene for PBD. $F_{a, 10 \mathrm{~L}}$ was calculated using eq. (18), and $\xi_{a} \eta_{a}$ was calculated from the mass of detected VOCs $m_{\mathrm{m}, \mathrm{de}}$ using eqs. (16) and (17). Although $\xi_{a} \eta_{a}$ was almost constant from 0.7 to 0.9 for $v_{\mathrm{de}}=3$ to $68 \mathrm{sccm}, F_{a, 10 \mathrm{~L}}$ decreased while $v_{\mathrm{de}}$ increased. We considered that the response time of $\Delta f_{a, 10 \mathrm{~L}}$ from VOC sensing might be an important parameter of this phenomenon because a small $v_{\text {de }}$ rendered $\Delta f_{a, 10 \mathrm{~L}}$ high. The lower the $v_{\text {de }}$ produced, the higher the $F_{a, 10 \mathrm{~L}}$; however, it made the width of the peaks (or deviation of Gaussian curve $\kappa_{a}$; we neglected $j$ when $j$ $=1$ ) large and gave poor separation capability among VOCs. Here, $\kappa_{a}$ was estimated by parameter fitting of the Gaussian curve using eq. (15) with $j=1$. Thus, we decided that an adequate $v_{\mathrm{de}}$ was $17.2 \mathrm{sccm}\left(2.8 \times 10^{-5} \mathrm{~m}^{3} / \mathrm{s}\right)$. In this case, the typical $\kappa_{a}$ was 12 to 13 $\mathrm{s}$ for four VOCs. Taking the $1 / v_{\mathrm{de}}$ dependence of $t_{\mathrm{Pa}}$ and using eq. (13), we can estimate the desorption temperature $T_{a}$ at $1 / v_{\mathrm{de}}=0$ for each VOC. Table 2 shows several important values estimated from the $v_{\mathrm{de}}$ dependence of $\Delta f_{a, 10 \mathrm{~L}}$, including $T_{a}, \xi_{a} \eta_{a}, F_{a, 10 \mathrm{~L}}, \kappa_{a}$, and $t_{\mathrm{Pa}}$ at $v_{\mathrm{de}}=17.2 \mathrm{sccm}$. The maximum $F_{a, 10 \mathrm{~L}}$ was 910 for acetone with $640 \mathrm{~nm}$ PAB and 2,520 $\mathrm{nm}$ PBD, and the minimum was 460 for p-xylene with 2,520 nm PBD. 


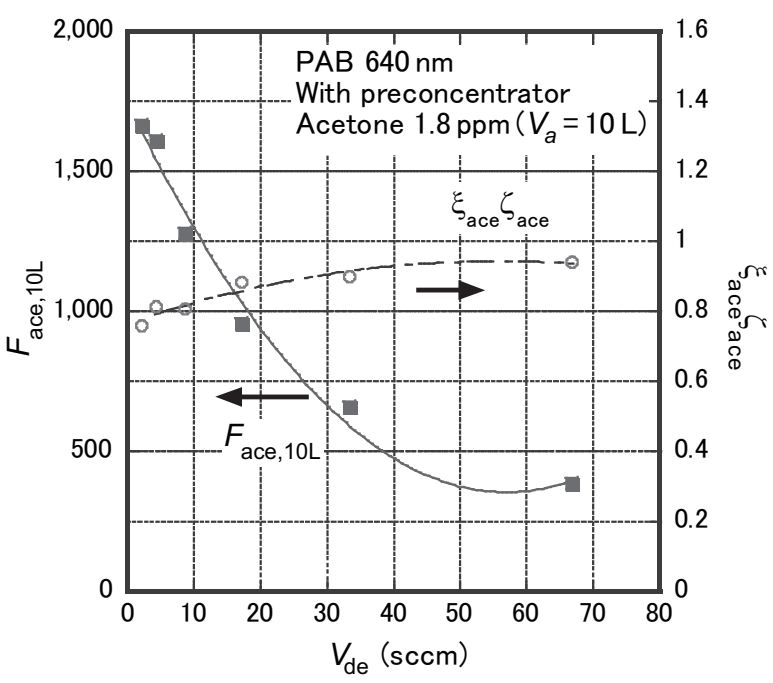

(a)

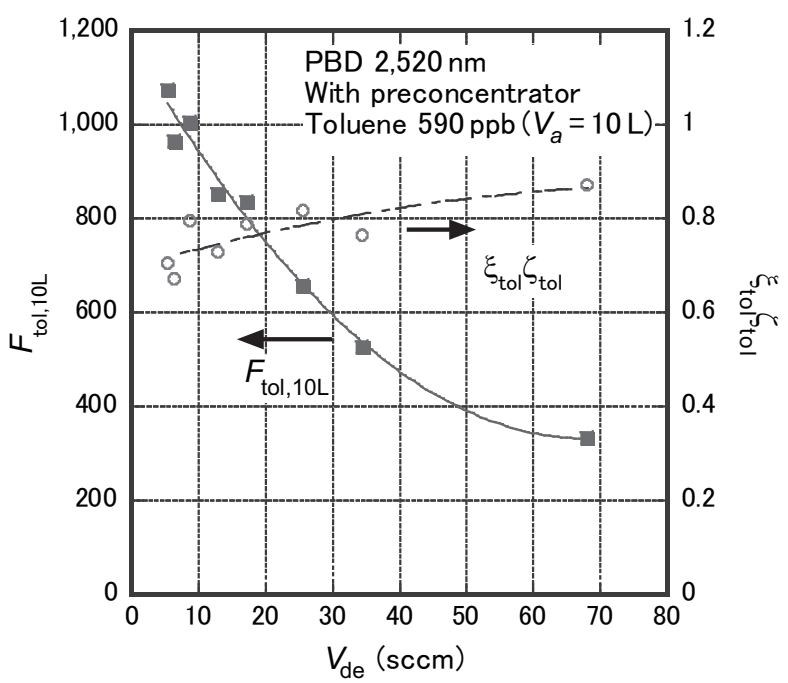

(b)

Fig. 12. Concentration factor and total system efficiency for our system: (a) acetone using PABcoated sensor, (b) toluene using PBD-coated sensor.

Table 2

System sensitivities, efficiency and detection limit for VOCs for PAB- and PBD-coated cantilevers.

\begin{tabular}{|c|c|c|c|c|c|c|c|}
\hline \multirow[t]{2}{*}{ Item } & \multirow[t]{2}{*}{ Unit } & \multicolumn{3}{|c|}{ PAB $640 \mathrm{~nm}$} & \multicolumn{3}{|c|}{ PBD 2,520 nm } \\
\hline & & Acetone & 1-Propanol & Toluene & Acetone & Toluene & p-Xylene \\
\hline$T_{a}$ & ${ }^{\circ} \mathrm{C}$ & 132 & 138 & 275 & 131 & 276 & 325 \\
\hline Sensitivity $s_{a}$ & $\mathrm{~Hz} / \mathrm{ppm}$ & 0.036 & 0.15 & 0.25 & 0.025 & 0.62 & 1.85 \\
\hline $\begin{array}{l}\text { System sensitivity } \\
S_{a, 10 \mathrm{~L}}\end{array}$ & $\begin{array}{l}\mathrm{Hz} / \mathrm{ppm} \\
\left(V_{a}=10 \mathrm{~L}\right)\end{array}$ & 32.7 & 88.5 & 148 & 22.7 & 514 & 850 \\
\hline $\begin{array}{l}F_{a, 10 \mathrm{~L}} \\
\left(v_{\mathrm{de}}=17.2 \mathrm{sccm}\right)\end{array}$ & $\left(V_{a}=10 \mathrm{~L}\right)$ & 910 & 590 & 590 & 910 & 830 & 460 \\
\hline $\begin{array}{l}\xi_{a} \eta_{a} \\
\left(v_{\mathrm{de}}=17.2 \mathrm{sccm}\right)\end{array}$ & & 0.88 & 0.53 & 0.6 & 0.8 & 0.78 & 0.45 \\
\hline $\begin{array}{l}\kappa_{a} \\
\left(v_{\mathrm{de}}=17.2 \mathrm{sccm}\right)\end{array}$ & $\mathrm{s}$ & 12.2 & 11.5 & 12.9 & 11.9 & 12.6 & 13 \\
\hline $\begin{array}{l}t_{\mathrm{Pa}} \\
\left(t_{0}=179 \mathrm{~s}, v_{\mathrm{de}}=17.2 \mathrm{sccm}\right)\end{array}$ & $\mathrm{s}$ & 327.5 & 345.7 & 456 & 320.6 & 446.6 & 493.1 \\
\hline Standard deviation $\lambda$ & $\mathrm{Hz}$ & 0.13 & 0.13 & 0.13 & 0.11 & 0.11 & 0.11 \\
\hline$D L_{a, 10 \mathrm{~L}}$ & $\begin{array}{l}\mathrm{ppb} \\
\left(V_{a}=10 \mathrm{~L}\right)\end{array}$ & 12 & 4.4 & 2.6 & 14.5 & 0.6 & 0.4 \\
\hline
\end{tabular}




\subsubsection{Estimation of system sensitivity}

To estimate the system sensitivity $S_{a}$ described by eq. (19), the $C_{a, 10 \mathrm{~L}}$ dependence of $\Delta f_{a, 10 \mathrm{~L}}$ was measured for the same samples as in the previous section. Figure 13 shows the $C_{a, 10 \mathrm{~L}}$ dependence of $\Delta f_{a, 10 \mathrm{~L}}$ of (a) the PAB-coated cantilever for acetone, 1-propanol, and toluene, and (b) the PBD-coated cantilever for toluene and p-xylene. The tangent of the straight line for each VOC was $S_{a, 10 \mathrm{~L}}$, and is listed in Table 2. The maximum $S_{a, 10 \mathrm{~L}}$ was $850 \mathrm{~Hz} / \mathrm{ppm}$ when $V_{a}=10 \mathrm{~L}$ for p-xylene with $2,520 \mathrm{~nm} \mathrm{PBD}$ and the minimum was 22.7 for acetone with 2,520 nm PBD.

As final results, we estimated the detection limit $D L_{a, 10 \mathrm{~L}}$ using eq. (20). A standard deviation $\lambda$ was calculated with 11 points (for $33 \mathrm{~s}$ ) of $\Delta f_{a}$ at the same time of measurement. The estimated values were $12 \mathrm{ppb}$ for acetone, $0.6 \mathrm{ppb}$ for toluene, and 0.4 $\mathrm{ppb}$ for $\mathrm{p}$-xylene, as shown in Table 2 .

\subsubsection{Separation of acetone and 1-propanol using two-Gaussian model}

We tried to estimate the concentrations of acetone and 1-propanol from a mixed gas sample by fitting of two Gaussian curves using a PAB $\left(t_{\text {Poly }}=640 \mathrm{~nm}\right)$-coated cantilever sensor. Since $T_{\text {ace }}$ (for acetone $)=132^{\circ} \mathrm{C}$ and $T_{\text {pnl }}$ (for 1 -propanol $)=138^{\circ} \mathrm{C}$ were similar, it was very difficult to distinguish the peaks on the response curve. Therefore, we used eq. (15) to explain the actual response curve, taking account of adequate fitting parameters. Although eq. (15) has three fitting parameters $\left(\Delta f_{\mathrm{Pa}, j}, t_{\mathrm{Pa}, j}\right.$, and $\left.\kappa_{a, j}\right)$ for each Gaussian function, $t_{\mathrm{Pa}, j}$ and $\kappa_{a, j}$ should be constant, and only $\Delta f_{\mathrm{Pa}, j}$ can be varied for estimating the quantitative value of $C_{a, 10 \mathrm{~L}}$. Figure 14 shows the fitting result to decompose two

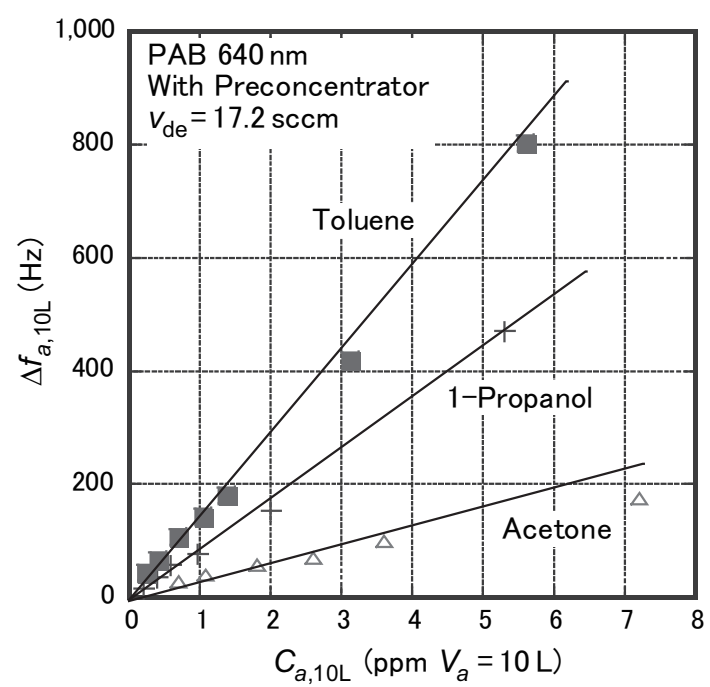

(a)

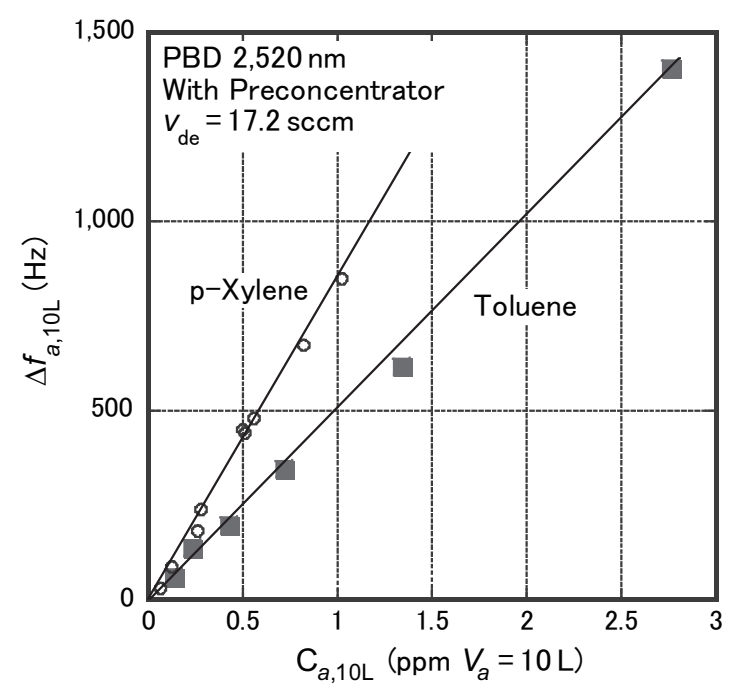

(b)

Fig. 13. Frequency shift of polymer-coated cantilever for four VOCs with preconcentrator: (a) 640-nm-thick PAB thin film, (b) 2,520-nm-thick PBD thin film. 


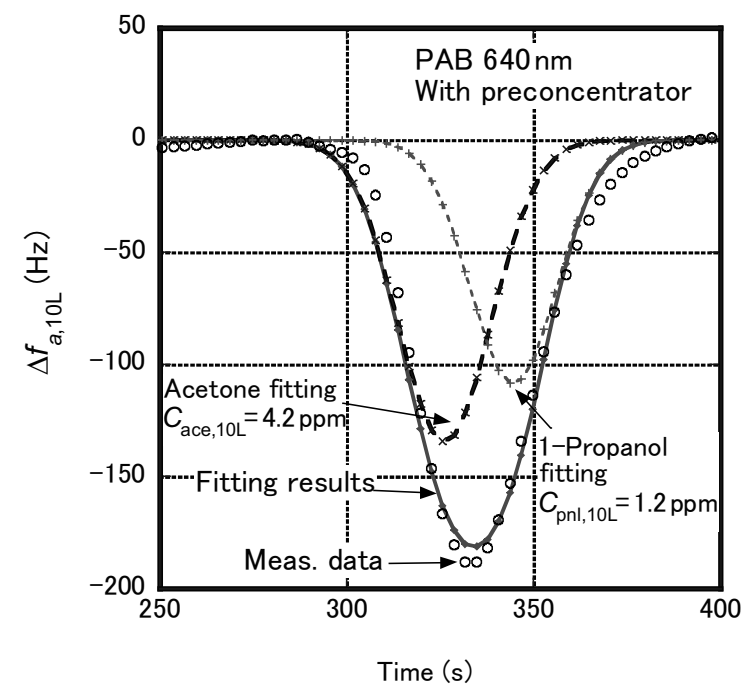

Fig. 14. Decomposition results using two Gaussian curve fittings with parameters shown in Table 2.

Gaussian curves for acetone and 1-propanol. The actual $C_{a, 10 \mathrm{~L}}$ values of mixed gas were $C_{\text {ace, } 10 \mathrm{~L}}=3.6 \mathrm{ppm}$ and $C_{\mathrm{pnl}, 10 \mathrm{~L}}=1.0 \mathrm{ppm}$, but the calculated values were 4.2 and $1.2 \mathrm{ppm}$, respectively. These discrepancies of $20 \%$ were inferred to be derived from the accuracy of $F_{a, 10 \mathrm{~L}}$ at low concentration.

\section{Discussion}

The design, structure and experimental results of a compact chemical sensor system employing polymer-coated multiple-cantilever-type mass sensors were presented. This sensor system had two merits for actual applications: (1) high sensitivity and (2) analytical function.

\subsection{High sensitivity}

High sensitivity was achieved using a preconcentrator of $6.35 \mathrm{~mm}$ diameter, an air pump with a large flow rate of $2.0 \mathrm{~L} / \mathrm{min}$, and a small sensor chamber of $0.3 \mathrm{cc}$. A 10 L sample of VOC was concentrated for $5 \mathrm{~min}$ and a $60 \mathrm{~L}$ volume sample for $30 \mathrm{~min}$ for practical application. This large sample volume made the concentration factor high. A textile structure of carbon fiber as the preconcentration material was also distributed to accomodate a large sample volume. The sensitivity, concentration factor, and detection limit for the $10 \mathrm{~L}$ toluene sample during $5 \mathrm{~min}$ of pumping were $514 \mathrm{~Hz} / \mathrm{ppm}$, and 830 and $0.6 \mathrm{ppb}$, respectively. For the $60-\mathrm{L}$ sample in $30 \mathrm{~min}$ pumping, they were $3,084 \mathrm{~Hz} / \mathrm{ppm}$, 
4,980 and $0.1 \mathrm{ppb}$, respectively. It is considered that the detection limit for actual application is 3 to 5 times larger than the estimated one. Since the indoor concentration guideline values of chemicals are $70 \mathrm{ppb}$ for toluene and $200 \mathrm{ppb}$ for xylene in Japan, ${ }^{(20)}$ these detection limits of our chemical sensor system are good enough for monitoring these environmental solvents in a room.

\subsection{Analytical function}

This system is capable of analysis by two methods: (a) measurement of peak delay time of sensor response caused by desorption temperature difference of heated preconcentrator and (b) by the use of multiple cantilever sensors with different polymers. Our system was capable of distinguishing toluene and p-xylene from the difference in peak times. Even though the peak times of the two VOCs were similar, the concentrations of the two were estimated by parameter fitting of multiple Gaussian functions from an actual response curve. We confirmed the decomposition of the acetone and 1-propanol mixed sample by these methods. A key to the operation of multiple cantilevers was to install circuits preset for resonance frequency and feedback phase at cantilever switching by MPX. Since the resonant frequency varied with the thickness of polymer and the feedback phase varied with the site of the cantilever in a chip, it was very difficult to achieve an adequate oscillation with multiple cantilevers with different thicknesses and different polymers.

In this work, we integrated eight microcantilevers including stress gauges and a $\mathrm{p}-\mathrm{n}$ diode as a temperature sensor on a chip, and four cantilevers among them were operated by multiplexing. Other electronic circuits consisted of discrete parts, operational amplifiers, ICs, and microcomputers. In the future, all electronic circuits will be integrable on the same silicon chip, as well as cantilevers, and will act as smart sensors with various intelligent functions. We also consider this system to be the first compact chemical sensor system using a MEMS mass-sensitive resonator for the purpose of environmental monitoring.

\section{Conclusions}

We developed a highly sensitive compact chemical sensor system employing a polymer-coated microcantilever sensor array and a thermal preconcentrator. The design, structure, and experiment results were reported. This sensor system had 1) a sub-ppb detection limit owing to concentration by a preconcentrator and 2) analytical functions enabled by thermal desorption of the preconcentrator and multiple cantilevers (acting as mass sensor) with different polymers. The preconcentration factor and system efficiency of sensing were estimated to be 830 and 0.78 , respectively, for toluene. The estimated detection limit of the sensor system was less than $1 \mathrm{ppb}$ for toluene and $\mathrm{p}$-xylene with a $10 \mathrm{~L}$ sample volume, which is good enough for application to environmental monitoring. The separate detection of the mixed toluene and p-xylene was also achieved in the form of different time peaks during the heating preconcentrator operation. 


\section{Acknowledgment}

This research was supported by the Regional Innovation Cluster Program of Nagano, through funds granted by the Ministry of Education, Culture, Sports, Science and Technology (MEXT), Japan.

\section{References}

1 M. Maute, S. Raible, F. E. Prins, D. P. Kern, H. Ulmer, U. Weimar and W. Goepel: Sens. Actuators, B 58 (1998) 505.

2 H. P. Langa, M. K. Ballera, R. Berger, C. Gerber, J. K. Gimzewski, F. M. Battiston, P. Fomaro, J. P. Ramseyer, E. Meyer and H. J. Giintherodt: Anal. Chim. Acta 393 (1999) 59.

3 D. Lange, C. Hagleitner, A. Hierlemann, O. Brand and H. Baltes: Anal. Chem. 74 (2002) 3084.

4 I. Dufour and L. Fadel: Sens. Actuators, B 91 (2003) 353.

5 T. Ikehara, J. Lu, M. Konno, R. Maeda and T. Mihara: J. Micromech. Microeng. 17 (2007) 2491.

6 J. Lu, T. Ikehara, Y. Zhang, R. Maeda and T. Mihara: Jpn. J. Appl. Phys. 45 (2006) 8795.

7 Y. Liu, T. Mihara, M. Kimura, M. Takasaki and T. Hirai: Proc. 24th Sensor Symp. (The Institute of Electrical Engineers of Japan, Tokyo, 2007) p. 309.

8 T. Mihara, T. Ikehara, J. Lu, R. Maeda, T. Fukawa, M, Kimura, Y. Liu and T. Hirai: Proc. 12th Int. Mtg. on Chemical Sensors (Sensor Division of the Electrochemical Society, Columbus Ohio, 2008) p. 533.

9 T. Mihara, T. Ikehara, J. Lu, R. Maeda, T. Fukawa and M. Kimura: Olfaction and Electronic Nose: Proc. 13th Int. Symp. (American Institute of Physics, Conference Proceedings, Brescia, 2009) p. 79.

10 T. Mihara, T. Ikehara, J. Lu, R. Maeda, T. Fukawa, M. Kimura, Y. Liu and T. Hirai: Proc. 25th Sensor Symp. (The Institute of Electrical Engineers of Japan, Okinawa, 2008) p. 591.

11 T. Mihara, T. Ikehara, M. Konno, R. Maeda, M. Kimura and T. Fukawa: IEEJ Trans. SM 130 (2010) 275 (in Japanese).

12 W. A. Groves, E. T. Zellersa and G. C. Fryec: Anal. Chim. Acta 371 (1998) 131.

13 F. Bender, N. Barie, G. Romoudis, A. Voigt and M. Rapp: Sens. Actuators, B 93 (2003) 135.

14 T. Nakamoto, K. Sukegawa and E. Sumitomo: IEEE Sens. J. 5 (2005) 68.

15 M. J. Henderson, B. A. Karger and G. A. Wrenshall: Diabetes 1 (1952) 188.

16 R. D. Blevins: Formulas for Natural Frequency and Mode Shape (Krieger Publishing, Malabar, 1979) Chapter 8, p. 108.

17 T. Ikehara, M. Konno, S. Murakami, T. Fukawa, M. Kimura and T. Mihara: Sens. Mater., 23 (2011) 381 .

18 P. A. Redhead: Vacuum 12 (1962) 203.

19 M. Kimura, Y. Liu, R. Sakai, S. Sato, T. Hirai, T. Fukawa and T. Mihara: Sens. Mater., 23 (2011) 419.

20 Guideline and standard evaluation method of the VOC indoor concentration in room: Japanese Ministry of Health, Labour and Welfare, Tokyo (2000) (in Japanese). 